\title{
PERDA DE PESO E CONSUMO DE BEBIDAS DURANTE SESSÕES DE TREINAMENTO DE BALLET CLÁSSICO
}

\author{
ESP. CLÁUDIA VILLELA DA SILVA \\ Nutricionista, Especialista em Nutrição Clínica \\ (Porto Alegre - Rio Grande do Sul - Brasil) \\ e-mail: claudinha_vi@hotmail.com
}

\author{
MS. ANA PAULA TRUSSARDI FAYH \\ Nutricionista e Educadora Física, Mestre em Ciências do Movimento Humano, \\ Docente dos cursos de Nutrição e Educação Física do Centro Universitário Metodista IPA \\ (Porto Alegre - Rio Grande do Sul - Brasil) \\ e-mail: apfayh@yahoo.com.br
}

\begin{abstract}
RESUMO
O objetivo do estudo foi analisar a perda de peso (PP) e o consumo de líquidos durante sessões de treinamento de ballet clássico. A amostra foi composta por vinte e uma bailarinas, que foram avaliadas em três sessões de ballet: uma sessão sem oferta de líquidos, uma sessão com oferta de água e outra com oferta de bebida esportiva. Na sessão em que as bailarinas receberam água, a mediana de consumo foi 376, $19 \mathrm{~mL}$ e a mediana de PP foi - 190g. Na sessão com bebida esportiva, a mediana do consumo foi de 226, $19 \mathrm{~mL}$ e a mediana da PP foi - I 50g. Observou-se diferença na ingestão de líquidos, mas não na PP, durante as sessões com consumo de líquidos ( $p=0,0$ l e $p=0,79$, respectivamente). As bailarinas, voluntariamente, beberam mais água do que bebida esportiva durante os treinos de ballet clássico.
\end{abstract}

PALAVRAS-CHAVE: Dança; hidratação; desidratação; sede. 


\section{INTRODUÇÃO}

O ballet clássico é um exercício físico caracterizado por movimentos de flexões e extensões, saltos e giros envolvendo velocidade e equilíbrio, além de requerer que o praticante possua graça, leveza e delicadeza. É denotado através do desenvolvimento de passos técnicos, harmoniosos e representações elaboradas (ACHCAR, 1998). Trata-se de uma manifestação artística exigente no critério anatômico corporal, a qual o bailarino deve apresentar uma figura magra e longilínea, almejando um baixo percentual de gordura, resultando no criterioso cuidado no controle de peso (BETANCOURT et al., 2007).

A adoção de dietas com baixo teor calórico, a ingestão de chás e o uso indevido de diuréticos e laxantes, são hábitos adotados por bailarinos, tal a busca obsessiva pela redução do peso corpóreo (OLIVEIRA et al., 2003). Entretanto, essas condutas são prejudiciais não somente no desempenho, mas na saúde do bailarino, promovendo notáveis alterações fisiológicas e funcionais (MORGAN; VECCHIATTI; NEGRÃO, 2002). Algumas destas práticas podem comprometer o estado de hidratação do indivíduo, repercutindo em um menor desempenho durante a dança. Como o ballet é caracterizado por movimentos suaves, os sinais e sintomas da desidratação prejudicam a execução adequada de alguns passos na coreografia (ACHCAR, 1998; MORGAN; VECCHIATTI; NEGRÃO, 2002).

Sendo o ballet clássico considerado uma modalidade esportiva, ocorre uma expressiva atividade músculo-esquelética, gerando gasto energético e aumento de calor corporal. Ao considerarmos as condições ambientais e a vestimenta utilizada durante a prática deste exercício, pode ocorrer o favorecimento da perda de líquidos corporais por meio da sudorese (CASA et al., 2000). Com isso, torna-se importante avaliar estratégias para repor os líquidos e eletrólitos perdidos durante esta atividade, como forma de prevenção a desidratação e hiponatremia no atleta, sendo estas prejudiciais ao seu desempenho e saúde (CASA et al., 2000; CARVALHO et al.,2003; LIMA; MICHELS; AMORIM, 2007).

A água é uma das principais fontes de hidratação, por apresentar baixo custo, ser amplamente disponível e possuir efeito de esvaziamento gástrico rápido (CASA et al., 2000). Entretanto, por não conter sódio em quantidades significativas e carboidratos, a hidratação com água durante os exercícios pode aumentar os riscos de desenvolvimento de hiponatremia e hipoglicemia respectivamente. Além disso, por ser insípida, tem por desvantagem não favorecer ao consumo voluntário, aumentando a probabilidade do desequilíbrio hidro-eletrolítico (CASA et al., 2000). Com isso, a utilização de repositores hidroeletrolíticos torna-se uma opção razoável 
durante a prática esportiva, pois oferece uma quantidade adequada de água, açúcares e eletrólitos, principalmente o sódio, devendo ser escolhidas principalmente em exercícios que tenham duração igual ou superior a uma hora (CARVALHO et al.,2003; LIMA; MICHELS; AMORIM, 2007; ARAGÓN-VARGAS et al., 200 I).

A literatura atual possui poucos estudos relacionados ao consumo, aceitação de líquidos e desidratação em profissionais da dança. Estudos prévios com outros esportes apontam que a desidratação é mais comum em atletas do sexo feminino (SALAS, 2006; UMEDA et al., 2004), bem como apontam que este público é mais vulnerável a alterações em sua termorregulação devido às suas características antropométricas e hormonais (CHEUVRONT; HAYMES, 200I). Com isso, torna-se importante conhecer a magnitude destas alterações fisiológicas durante a prática da dança. Diante destas considerações, o presente estudo objetiva verificar a ingestão de água e repositor hidroeletrolítico durante sessões de treinamento de ballet clássico, bem como quantificar a perda de peso ocasionada nas sessões com e sem a ingestão de líquidos.

\section{MÉTODOS}

\section{CARACTERIZAÇÃO DA POPULAÇÃO E DA AMOSTRA}

Foram avaliadas vinte uma bailarinas do sexo feminino de uma Escola de Dança de Porto Alegre (Rio Grande do Sul, Brasil), com idade entre dezoito e trinta anos. Como critérios de inclusão, as bailarinas deveriam ter mais de sete anos de prática neste tipo de dança e um volume de treino de pelo menos quatrocentos e cinquenta minutos por semana. A totalidade de bailarinas da Escola de Dança, com este perfil, foi convidada a participar do estudo a partir da exposição do projeto de pesquisa, previamente aprovado pelo comitê de Ética e Pesquisa da Rede Metodista de Educação do Sul (033/2008), e todas aceitaram participar mediante a assinatura do Termo de Consentimento Livre e Esclarecido, em duas vias.

\section{DESCRIÇÃO DE COLETA E ANÁLISE DE DADOS}

Inicialmente, todas as voluntárias realizaram avaliação antropométrica antes da sessão de treino. Para o registro da massa corporal, utilizou-se uma balança digital (Plenna ${ }^{\circledR}$ ), e para a medida da estatura utilizou-se uma fita métrica (Sanny $\left.{ }^{\circledR}\right)$, a qual foi fixada em uma parede, sem rodapé, com limite inferior da fita no chão e o superior a 200 cm do mesmo. Cada bailarina permaneceu em pé, ereta, com os braços estendidos ao longo do corpo e com a cabeça erguida, posicionada centralmente à fita métrica, com os cabelos soltos, calcanhares encostados à parede, assim como 
ombros e nádegas. Após, foi colocada um esquadro sobre o vértex da voluntária, fixando-a contra a cabeça, para que pudesse ser feita a leitura da estatura medida. A partir dos valores encontrados (peso e estatura), foi realizado o cálculo do Índice de Massa Corporal (IMC) $\left(\mathrm{kg} / \mathrm{m}^{2}\right)$, que foi classificado conforme as recomendações da Organização Mundial da Saúde para adolescentes (bailarinas com idade inferior a 20 anos) e para adultos (quando a idade era igual ou superior a vinte anos) WORLD HEALTH ORGANIZATION, 1995, 2007).

Para a medida das dobras cutâneas, utilizou-se um adipômetro científico (Cescorf®), com precisão de 0, I mm. Foram medidas as seguintes dobras cutâneas, do lado direito do corpo de cada bailarina: subescapular, triciptal, peitoral, axilar média, supra-ilíaca, abdominal e coxa. A ordem das medidas realizou-se de forma rotacional, e o cálculo do percentual de gordura seguiu o protocolo proposto por Jackson, Pollock e Ward (1980), para atletas do sexo feminino. Para a classificação da adiposidade corporal, observou-se as recomendações de adiposidade corporal de bailarinas segundo Heyward e Stolarczyk (1996), que considera adequado valores entre 13 a $20 \%$.

Após a realização da avaliação antropométrica, as bailarinas receberam orientações para a realização da coleta dos dados, como não se alimentar em um período de tempo inferior a duas horas dos testes, não ingerir líquidos a menos de uma hora dos testes e urinar 30 minutos antes de cada teste. Estas orientações foram reforçadas através de contato telefônico e pessoal pela pesquisadora no dia do teste. Foram agendadas três novas datas para a realização do protocolo experimental, que ocorreu na própria sede da Escola de Ballet. A ordem dos testes ocorreu de forma randomizada através de sorteio prévio.

As sessões experimentais com as bailarinas consistiram em treinos habituais ballet clássico, com duração de 90 minutos. Estas se caracterizaram por idêntica ordem de movimentos corporais e esforços físicos. Em todos os momentos, ocorreu o controle da temperatura ambiente da sala de treinos com auxílio de um termômetro (Incortem ${ }^{\circledR}$ ), que oscilou entre 20,9 e $22^{\circ} \mathrm{C}$. Houve também a medida da massa corporal das voluntárias nos momentos imediatamente antes e imediatamente depois da sessão de treino estando as voluntárias vestindo apenas roupas íntimas. Em uma das sessões, com o objetivo de avaliar a perda de líquidos durante o treinamento de ballet clássico, as bailarinas receberam a orientação de não beber nenhum tipo de líquido durante toda a aula. Nas outras duas sessões, foram disponibilizadas às bailarinas duas garrafas de $500 \mathrm{~mL}$ de água mineral sem gás da marca ljuíß e duas garrafas de $500 \mathrm{~mL}$ de repositor hidroeletrolítico (Gatorade ${ }^{\circledR}$ ), sendo um tipo de bebida para cada sessão. Ao final destas sessões, houve o recolhimento de todas as garrafas de bebidas oferecidas, verificando-se a quantidade ingerida por cada bailarina. Igualmente, foram 
medidas a massa corporal nos momentos antes e depois da sessão de treino. Para o cálculo da perda de peso, calculou-se o percentual de variação do peso do início para o final da sessão de treino (peso final (kg)-peso inicial (kg)/l 00).

\section{ANÁLISE ESTATÍSTICA}

Os dados foram estruturados e analisados utilizando o pacote estatístico SPSS (Statistical Package for Social Sciences) versão 17.0 para Windows. Os resultados foram expressos em médias e desvios padrão para os dados de caracterização da amostra (idade, medidas antropométricas e percentual de desidratação), e em mediana e valores mínimos e máximos para os dados de perda de peso e consumo de bebida. Para comparar o consumo de líquidos durante as sessões de treino, foi utilizado o teste de Wilcoxon, e para comparar a perda de peso durante as três sessões de treino, foi utilizado o teste de Friedman. $\bigcirc$ nível de significância aceito para este estudo foi de $5 \%$.

\section{RESULTADOS}

Os dados de caracterização da amostra estão descritos na tabela I , que aponta que as bailarinas apresentaram valores médios de IMC e percentual de gordura adequado para esta modalidade esportiva. O percentual de perda de peso da sessão sem a ingestão de líquidos demonstra que a sessão de treino foi caracterizada por um baixo percentual de desidratação, com valor inferior a $2 \%$.

Tabela I. Dados de Caracterização da Amostra.

\begin{tabular}{ll}
\hline & Média $\pm \mathrm{dp}$ \\
\hline Idade (anos) & $21,29 \pm 2,53$ \\
Massa Corporal $(\mathrm{kg})$ & $55,19 \pm 5,37$ \\
Estatura $(\mathrm{cm})$ & $164,05 \pm 5,88$ \\
IMC $(\mathrm{kg} / \mathrm{m} 2)$ & $20,64 \pm 1,25$ \\
Percentual de gordura (\%) & $19,20 \pm 4,38$ \\
Percentual de perda de peso (\%)* & $-0,6425 \pm 0,4939$ \\
\hline
\end{tabular}

* Perda de perda peso durante a sessão de treinamento de 90 minutos sem a ingestão de bebidas.

A Tabela 2 demonstra os valores de medianas, os valores mínimos e máximos do consumo das bebidas e da perda de peso nas sessões de treinamento. Observouse que as bailarinas possuem maior aceitação de água durante a sessão de treino em relação à bebida esportiva. Entretanto, mesmo com o consumo superior de água, a perda de peso durante a sessão não foi estatisticamente diferente da sessão com bebida esportiva $(p=0,79)$. Quando analisamos a perda de peso nas três sessões, 
incluindo a sessão sem o consumo de bebida, pode-se perceber que a perda de peso é superior quando as bailarinas não realizam a ingestão de líquidos.

Tabela 2. Comparação entre o consumo das bebidas e a perda de peso durante as sessões de treinamento.

\begin{tabular}{lcccc}
\hline & Mediana & Valor mínimo & Valor máximo & P \\
\hline Consumo de Água $(\mathrm{mL})$ & 400 & 50 & 600 & \\
$\begin{array}{l}\text { Consumo de Bebida Esportiva }(\mathrm{mL}) \\
\text { Perda de peso sessão com ingestão }\end{array}$ & 200 & 50 & 500 & 0,0 I* \\
$\begin{array}{l}\text { de água (g) } \\
\begin{array}{l}\text { Perda de Peso sessão com ingestão de } \\
\text { bebida esportiva (g) }\end{array}\end{array}$ & -300 & -500 & +200 & \\
$\begin{array}{l}\text { Perda de Peso sessão sem ingestão } \\
\text { de líquidos (g) }\end{array}$ & -300 & -400 & +800 & \\
\hline
\end{tabular}

* valor de p obtido com o teste de Wilcoxon

** valor de p obtido através do teste de Friedman

\section{DISCUSSÃO}

O principal achado deste estudo é que as bailarinas, de forma voluntária, ingeriram mais água do que bebida esportiva quando ambas foram oferecidas na mesma quantidade durante uma sessão de treino. Este é um estudo pioneiro para verificar esta aceitação, e com isso essa limitação torna-se presente durante a discussão dos resultados. Outros aspectos importantes a serem mencionados são o pequeno tamanho da amostra, que não nos permite uma generalização dos resultados, bem como os aspectos relacionados ao consumo de água de uma população residente abaixo do trópico de capricórnio, onde oscilações climáticas nem sempre favorecem um grande consumo de líquidos. Entretanto, os dados do presente estudo tornam-se importantes, pois contribuem com a literatura científica na caracterização do consumo hídrico e perda líquida em bailarinas clássicas.

Em relação ao estado nutricional avaliado pelo IMC, em média, as bailarinas da amostra estavam eutróficas, sendo apenas uma integrante classificada como desnutrida de grau leve, conforme classificações da World Health Organization ( 1995). Este perfil assemelha-se à pesquisa realizada com quatorze atletas de ginástica artística, com idades entre onze e quinze anos, que demonstrou que a maioria das atletas eram eutróficas (AZEVEDO; RIBEIRO, 2007). Quando nossos resultados são comparados com um estudo realizado com bailarinos profissionais do ballet nacional de Cuba (BETANCOURT; ALBIZU-CAMPOS; DÍAZ, 2007), percebemos 
a média de IMC deste estudo é inferior à nossa amostra, demonstrando que, quanto maior o grau de especialização no ballet, mais desejável torna-se um corpo mais longelíneo. Enfatiza-se que as bailarinas cubanas são atletas profissionais, e por essa razão possuem uma exigência maior no controle de peso, em relação às bailarinas deste estudo.

Grego et al. (2006), realizaram um estudo comparando o estado nutricional de jovens do sexo feminino praticantes de diferentes modalidades de dança. Os autores constataram que média do IMC foi menor no grupo das bailarinas clássicas ( I 8,25 $\left.\pm 2,04 \mathrm{~kg} / \mathrm{m}^{2}\right)$ quando comparado às bailarinas não clássicas $\left(20,74 \pm 1,95 \mathrm{~kg} / \mathrm{m}^{2}\right)$. Contudo, o IMC possui limitações quando aplicados em esportistas, pois não é capaz de oferecer informações da composição corporal do indivíduo, revelando uma baixa sensibilidade na identificação da distribuição de gordura (SIANI et al., 2002; GARN; LEONARD; HAWTHORNE, 1986).

A busca de um modelo corporal longelíneo, alongado e magro para o ballet clássico, a fim de proporcionar um melhor desempenho esportivo, acarreta em uma preocupação excessiva com a imagem física, com rígidos controles de peso e percentual de gordura corporal (JONES; THOMPSON; MALINA, 2002). Prati e Prati (2006), em avaliação do percentual de gordura de I I bailarinas clássicas, observaram que o perfil corporal das participantes está adequado, para esta modalidade de dança, por apresentarem um percentual de gordura médio de $22,7 \%$. Salientando que estar ou ser leve, trata-se de um benefício para o desenvolvimento da técnica clássica. Entretanto, Betancourt, Aréchiga e Ramirez (2008), ressaltam que uma bailarina que apresenta um percentual de gordura pequeno e um volume corporal maior pode ser considerado acima do peso no campo da dança. Torna-se relevante ressaltar que os valores de percentual de gordura considerados desejáveis para bailarinas são 13\% a 20\%, enquanto que para a população em geral do sexo feminino se esperam valores entre $20 \%$ a 30\% (HEYWARD; STOLARCZYK, 1996).

Existem basicamente três formas de avaliar a desidratação induzida por exercícios: pela variação do peso corporal durante a sessão, pela avaliação da osmolalidade plasmática ou pela gravidade específica e coloração da urina (CASA et al., 2000) No presente estudo, optou-se por avaliar a desidratação pela perda de peso durante as sessões de treinamento de ballet, pela facilidade de obtenção das medidas e aplicabilidade na prática esportiva. Em pesquisa nos sites de busca de artigos científicos, não foram encontrados estudos que avaliassem a perda de peso durante uma sessão de ballet clássico. As bailarinas da nossa amostra obtiveram um baixo percentual de desidratação, representando em média menos de $1 \%$ de perda líquida. Perella, Noriyuki e Rossi (2005), avaliaram a perda hídrica durante um treino intenso de rugby, demonstrando valores de perda de peso próximos ao presente estudo (média de I ,5\%). Os autores apontam que, mesmo sem um nível de desidratação considerado importante, devemos conscientizar os atletas acerca da importância da 
hidratação adequada. Mesmo uma leve desidratação (um por cento da massa corporal) pode-se ter aumento do esforço cardiovascular, ocasionado aumento desproporcional da frequência cardíaca durante o exercício, além de limitar a capacidade corporal de transferir calor dos músculos em contração para a superfície da pele, onde pode ser dissipado para o ambiente (MACHADO-MOREIRA et al., 2006).

A perda hídrica pelo suor em função da atividade física deverá resultar em ingestão de líquidos que, em média, levará entre 2 a 4 horas para equilibrar o nível citoplasmático dos tecidos corporais. Em exercícios mais prolongados, o nível de perda de líquidos pode aumentar, como no caso do estudo realizado com 89 triatletas (MARA et al., 2007), que exercitaram-se em média por 13 horas durante uma prova de Iron Man. Os resultados apontaram que $56 \%$ dos atletas desidratam até $3 \%$ durante a prova, e que $32,6 \%$ desidratam até $6 \%$, mesmo com reposição espontânea de líquidos. Nestes casos, a importância da hidratação adequada no intuito de evitar a ocorrência de sintomas moderados e graves de desidratação deve ser ressaltada.

Em relação ao consumo de líquidos, os dados demonstraram que as bailarinas, durante o treino, consomem de forma voluntária mais água do que bebidas esportivas. Nossos resultados divergem de outros estudos encontrados na literatura, que demonstram que atletas e praticantes de exercícios aceitam mais bebidas esportivas durante o exercício (KLEINER, 1999; PASSE; HORN; MURRAY, 2000). Estudo realizado com atletas do sexo feminino, à respeito da ingestão de bebidas com carboidratos, demonstrou que o conteúdo energético da bebida não influenciou no consumo voluntário durante a pratica esportiva (MINEHAN; RILEY; BURKER, 2002). Divergindo destes dados, as bailarinas do presente estudo, que apresentam uma preocupação estética exigida pela dança (BETANCOURT et al., 2007) tiveram maior aceitabilidade de água durante o exercício, provavelmente por ser um líquido hidratante ausente em calorias. Também existem outros fatores que interferem na escolha da natureza do líquido a ser consumido durante o exercício. A sede pode fazer com que atletas e esportistas ingiram uma quantidade muito maior de água do que de bebidas esportivas, que possuem um poder de saciedade aumentado devido à sua osmolalidade (CASA et al., 2000). Outro fato que deve ser levado em consideração diz respeito à adição de carboidrato na bebida, que retarda o processo de esvaziamento gástrico, fazendo com que os indivíduos consumam uma menor quantidade para evitar a sensação de plenitude (MACHADO-MOREIRA et al., 2006). Com isso, estudos adicionais que elucidem os motivos da escolha de determinada bebida para reidratação tornam-se necessários.

\section{CONCLUSÃO}

Nossos dados demonstraram que o treinamento da modalidade do ballet clássico apresenta um baixo nível de desidratação, visualizado através da perda de 
peso durante a sessão sem ingestão de líquidos. Quanto ao consumo de líquidos durante as sessões de ballet clássico, as bailarinas ingerem voluntariamente uma maior quantidade de água do que de bebidas esportivas durante a prática da modalidade. Este resultado vai contra as recomendações nutricionais aplicadas para esportes com duração superior a 60 minutos, e com isso atletas e treinadores precisam ficar atentos para evitar prejuízos à saúde. No entanto, este estudo se limita a fazer uma descrição da amostra estudada, ressaltando a necessidade de outros estudos serem realizados com este público, a fim de complementar as informações relacionadas ao tema abordado.

\section{Weight loss and beverage consumption during training sessions of classical ballet}

ABSTRACT: The purpose of this study was to analyze the weight loss(WL) and consumption fluids during training sessions in classical ballet. The sample was composed of 21 ballet dancers, who were evaluated in three sessions of ballet: session without a supply of liquid, a session with water supply and other with provision of sports drink. At the session in which the dancers received water, the median consumption was 376,19 mL and the median was WL-190g. In the session with sports drink, the median consumption was $226,19 \mathrm{~mL}$ and the median was WL-150g. Observed difference in fluid intake, but not in WL, during the sessions with fluid intake $(p=0,01$ and $p=0,79$, respectively). The dancers, voluntarily, drank more water than the sports drink during training of classical ballet.

KEYWORDS: Dancing; fluid therapy; dehydration; thirst.

\section{Pérdida de peso y el consumo de bebidas durante los períodos de sesiones de entrenamiento de ballet clasico}

RESUMEN: El objetivo de este estudio fue analizar la pérdida de peso (PP) y la ingesta de líquidos durante las sesiones de formación de ballet clásico. La muestra estuvo conformada por 2 I bailarines, que fueron evaluados en tres sesiones de ballet: sesión sin un suministro de líquido, una sesión com La prestación de agua y otro con bebida deportiva. En la sesión en la que los bailarines recibieron água, la mediana del consumo era de 376, 19 ml y la mediana de PP-190g. En la sesión con bebida deportiva, la mediana del consumo era de 226, $19 \mathrm{ml}$ y la mediana de PP-150g. Observado diferencia en la ingesta de líquidos, pero no en PP, durante las sesiones con la ingesta de líquidos ( $p=0,01$ y $p=0,79$, respectivamente). Los bailarines, voluntariamente, bebió más agua que bebida deportiva durante el entrenamiento del ballet clásico.

PALABRAS CLAVE: Baile; fluidoterapia; deshidratación; sed. 


\section{REFERÊNCIAS}

ACHCAR, D. Balé uma Arte. Rio de Janeiro: Ediouro, 1998.

ARAGÓN-VARGAS, L. F. et al. O consenso: atividade física no calor: regulação térmica e hidratação. In: . Cidade do México: Gatorade Sports Science Institute, 1999. Disponível em: http://www.dtreino.com.br/hidratacao.pdf. Acesso em: 01 jul. 201 I.

AZEVEDO, B. A. R; RIBEIRO, S.M.L. Avaliação do estado nutricional e do balanço energético de um grupo de atletas de ginástica artística. Motriz, Rio Claro, v. I3, n.3, p. I65-173, jul./set. 2007.

BETANCOURT, H. et al. Composición corporal de bailarines adolescentes de la Escuela Nacional de Ballet de Cuba. Antropo (online), v. I5, p. 23-33, 2007. Disponível em: < http:// www.didac.ehu.es/antropo/I5/I5-3/Betancourt.pdf>. Acesso em: 0 I jul. 201 I.

BETANCOURT, H; ALBIZU- CAMPOS, J. C; DÍAZ, M. E. Composición Corporal de bailarines élites de la compañía Ballet Nacional de Cuba. Rev.Cub. Alim. Nutr., Ciudad de La Habana, v. 17, n. I, p. 8-22, jan/jul. 2007.

BETANCOURT, H; ARÉCHIGA, J; RAMÍREZ, C. M. Estudio bioantropológico del concepto "gordura-delgadez" em un grupo de bailarines de ballet adolescentes de Cuba. Rev Nutr Clin, v. 10, n. 2, p. 56-62, 2008.

CARVALHO, T. et al. Diretriz da Sociedade Brasileira de Medicina do Esporte: modificações dietéticas, reposição hídrica, suplementos alimentares e drogas: comprovação de ação ergogênica e potenciais riscos para a saúde. Rev. Bras. Med. Esporte, Niterói, v. 9, n. 2, p. 43-56, mar./abr. 2003.

CASA, D. J. et al. National Athletic Trainer's Association Position Statement (NATA): fluid replacement for athletes. J Athletic Training, Columbus, v. 35, n. 2, p. 212-224, jun. 2000.

CHEUVRONT, S. N; HAYMES, E. M. Thermoregulation and marathon running: biological and environmental influences. Sports Med, Massachusetts, v.31, n. I 0, p.743-762, out. 2001 .

GARN, S. M; LEONARD, W. R; HAWTHORNE, V. M. Three limitations of the body mass index. Am J Clin Nutr, Bethesda, v. 44, p. 996-997, dez. 1986.

GREGO, L. G. et al. Aptidão física e saúde de praticantes de dança e de escolares. Salusvita, Bauru, v. 25, n. 2, p. 81-96, maio/ago. 2006.

HEYWARD, V. H; STOLARCZYK, L. M. Applied Body Composition Assessment. Champaign: Human Kinetics, 1996.

JACKSON, S. A; POLLOCK, M. L; WARD, A. Generalized Equations for predicting body density for women. Med Sci Sports Exerc, Indianapolis, v. 12, p. 175-182, fev. 1980. 
JONES, A. D. B; THOMPSON, A. M; MALINA, R.M. Growth and maturation in elite young female athletes. Sports Med Arthroscopy Review, San Francisco, v. I0, n. I, p. 42-49, mar. 2002.

KLEINER, S. M. Water: an essential but overlooked nutrient. J Am Diet Assoc, Philadelphia, v. 99, n.2, p. 200-206, fev. 1999.

LIMA, C; MICHELS, M. F; AMORIM, R. Os diferentes tipos de substratos utilizados na hidratação do atleta para melhora do desempenho. Rev. Bras. Nut. Esportiva, São Paulo, v. I, n. I, p. 73-83, jan./fev. 2007.

MACHADO-MOREIRA, C. A. et al. Hidratação durante o exercício: a sede é suficiente? Rev Bras Med Esporte, Niterói, v. 12, n. 6, p. 4 05-409, nov./dez. 2006.

MARA, L.S. et al. Alterações hidroeletrolíticas agudas ocorridas no Triatlon Ironman Brasil. Rev Bras Med Esporte, Niterói, v. I3, n. 6, p. 397-40I, nov./dez. 2007.

MINEHAN, M. R; RILEY, M. D; BURKER, L. M. Effect of flavour and awareness of kilojoule content of drinks on preference and fluid balance in team sports. Int J Sport Nutr. Exerc. Metabolism, Champaign, v. 12, n. I, p. 81-92, mar. 2002.

MORGAN, C.M; VECCHIATTI, I. R; NEGRÃO, A. B. Etiologia dos transtornos alimentares: aspectos biológicos, psicológicos e sócio-culturais. Rev. Bras. Psiquiatr, São Paulo, v. 24, n. 3, p. 18-23. dez. 2002.

OLIVEIRA, F. P. et al. Comportamento alimentar e imagem corporal em atletas. Rev Bras Med Esporte, Niterói, v. 9, n. 6, p.348-356, nov./dez. 2003.

PASSE, D. H; HORN, M; MURRAY, R. Impact of beverage acceptability on fluid intake during exercise. Appetite, Amsterdam, v. 35, n. 3, p. 219-229, dez. 2000.

PERELLA, M. M; NORIYUKI, P. S; ROSSI, L. Avaliação da perda hídrica durante treino intenso de rugby. Rev. Bras. Med. Esporte, Niterói, v. I I, n. 4, p. 229-232, jul./ago. 2005.

PRATI, S. R. A; PRATI, A. R. C. Níveis de aptidão física e análise de tendências posturais em bailarinas clássicas. Rev Bras Cineantropom Desempenho Hum, Florianópolis, v. 8, n. I, p.80-87, abr. 2006.

SALAS, C. A. M. Depleción hídrica en atletas escolares de taekwondo. Rev Intern Med Cien Activ. Física y Deporte, Habana, v. 6, n. 21, p. 29-37, mar. 2006.

SIANI, A. et al. The relationship of waist circumference to blood pressure: The Olivetti Herat Study. Am J Hypertens, New York, v. I5, p. 780-786, set. 2002.

UMEDA, T. et al. Effects of gender-related weight reduction on the physical condition of male and female college judoists. Japanese Journal of Hygiene, Aoba, v. 59, n. 9, p. 326-334, jul. 2004. 
WORLD HEALTH ORGANIZATION. Physical Status: The Use and Interpretation of Anthropometry. Report of a WHO Expert Commitee. WHO Report Series 854. Geneva: WHO, 1995. Disponível em: http://www.who.int/childgrowth/publications/physical_status/en/index. html. Acesso em: 01 de jul. 201 l.

WORLD HEALTH ORGANIZATION. Physical Status Development of a WHO growth reference for school-aged children and adolescents. Bulletin of the World Health Organization, Geneva, v. 85, n. 9, p. 660-667, set. 2007.

Recebido: 13 dez. 2010 Aprovado: 3 I maio 20 I I

Endereço de correspondência Cláudia Villela da Silva Av. Mariland 12/2/202 Bairro Auxiliadora. Porto Alegre, RS, Brasil. CEP: 90440-190. Telefone: (5I)3388-5687. 